\title{
Emissivity and conductivity of parton-hadron matter
}

\author{
O. Linnyk ${ }^{1}$, a E.L. Bratkovskaya ${ }^{2}$, W. Cassing ${ }^{1}$, V.P. Konchakovski ${ }^{1}$, and V. Ozvenchuk ${ }^{3}$ \\ ${ }^{1}$ Institut für Theoretische Physik, Universität Giessen, 35392 Giessen, Germany \\ ${ }^{2}$ Institut für Theoretische Physik, Johann Wolfgang Goethe Universität, 60438 Frankfurt am Main, Germany \\ Frankfurt Institute for Advanced Studies, 60438 Frankfurt am Main, Germany \\ ${ }^{3}$ SUBATECH, UMR 6457, Laboratoire de Physique Subatomique et des Technologies Associées, University \\ of Nantes - IN2P3/CNRS - Ecole des Mines de Nantes, 44072 Nantes Cedex 03, France
}

\begin{abstract}
We investigate the properties of the QCD matter across the deconfinement phase transition. In the scope of the parton-hadron string dynamics (PHSD) transport approach, we study the strongly interacting matter in equilibrium as well as the out-of equilibrium dynamics of relativistic heavy-ion collisions. We present here in particular the results on the electromagnetic radiation, i.e. photon and dilepton production, in relativistic heavy-ion collisions and the relevant correlator in equilibrium, i.e. the electric conductivity. By comparing our calculations for the heavy-ion collisions to the available data, we determine the relative importance of the various production sources and address the possible origin of the observed strong elliptic flow $v_{2}$ of direct photons.
\end{abstract}

The electromagnetic emissivity of strongly interacting matter is a subject of longstanding interest and is explored in particular in relativistic nucleus-nucleus collisions, where the photons (and dileptons) measured experimentally provide a time-integrated picture of the collision dynamics. The recent observation by the PHENIX Collaboration [1] that the elliptic flow $v_{2}\left(p_{T}\right)$ of 'direct photons' produced in minimal bias $\mathrm{Au}+\mathrm{Au}$ collisions at $\sqrt{s_{N N}}=200 \mathrm{GeV}$ is comparable to that of the produced pions was a surprise and in contrast to the theoretical expectations and predictions [2-6]. We have investigated the spectra and elliptic flow of dileptons and photons from relativistic heavy-ion collisions in the scope of the covariant transport approach Parton-Hadron String Dynamics (PHSD) in Refs. [7-11]. The PHSD model $[12,13]$ is an off-shell transport approach that consistently describes the full evolution of a relativistic heavy-ion collision from the initial hard scatterings and string formation through the dynamical deconfinement phase transition to the quark-gluon plasma as well as hadronization and the subsequent interactions in the hadronic phase. The two-particle correlations resulting from the finite width of the parton spectral functions are taken into account dynamically in the PHSD by means of the generalized off-shell transport equations [14] that go beyond the mean field or Boltzmann approximation [15]. The transport theoretical description of quarks and gluons in the PHSD is based on the Dynamical Quasi-Particle Model (DQPM) for partons that is constructed to reproduce lattice QCD (1QCD) results for the entropy density, energy density and pressure as functions of temperature for the quark-gluon plasma in thermodynamic equilibrium. In the hadronic sector, PHSD is equivalent to the Hadron-String Dynamics (HSD) approach [16-18]. For details about the DQPM model and the off-shell transport approach we refer the reader to the review Ref. [15].

\footnotetext{
ae-mail: olena.linnyk@theo.physik.uni-giessen.de
} 


\section{Electric conductivity}

The photon emission rate from the QGP or the hadronic system is controlled by the electric conductivity $\sigma_{0}$. The electric conductivity of hot QCD matter at various temperatures $T$ was studied within the PHSD approach for interacting partonic/hadronic systems in a finite box with periodic boundary conditions in Ref. [19] by computing the response of the strongly-interacting system in equilibrium to an external electric field. We found a sizeable temperature dependence of the ratio $\sigma_{0} / T$. The actual results for the ratio $\sigma_{0} / T$ versus the scaled temperature $T / T_{c}$ are displayed in Fig. 1 (left hand side) by the full round symbols. We observe a decreasing ratio $\sigma_{0} / T$ with $T / T_{c}$ in the hadronic phase, a minimum close to $T_{c}$ and an approximately linear rise with $T / T_{c}$ above $T_{c}(=158 \mathrm{MeV})$. The conductivity in the partonic phase from PHSD can be described by

$$
\frac{\sigma_{0}(T)}{T} \approx 0.01+0.16 \frac{T-T_{c}}{T_{c}}
$$

for $T_{c} \leq T \leq 2.2 T_{c}$. The 1QCD results [20-24] are represented in Fig. 1 (left hand side) by symbols with error bars (using $C_{E M}=2 e^{2} / 3, e^{2}=4 \pi \alpha, \alpha=1 / 137$ ). The linear rise with temperature is supported by the recent lattice results from Ref. [25] (not explicitly presented in this plot). In view of the pQCD prediction of a constant asymptotic value for $\sigma_{0} / T \approx 5.9769 / e^{2} \approx 65$ in leading order of the coupling [20,26], such a linear rise of the ratio with temperature might be surprising, but it can be understood in simple terms within the relaxation time approach with the DQPM interaction width as demonstrated in Ref. [19]. Our findings imply that the QCD matter even at $T \approx T_{c}$ is a much better electric conductor than $\mathrm{Cu}$ or $\mathrm{Ag}$ (at room temperature).

\section{Photon production in heavy-ion collisions}

In Ref [11] we have applied the PHSD approach to photon production in $\mathrm{Au}+\mathrm{Au}$ collisions at $\sqrt{s_{N N}}=200 \mathrm{GeV}$ and studied the transverse momentum spectrum and the elliptic flow $v_{2}$ of photons from hadronic and partonic production channels. We found that the PHSD calculations reproduce the transverse momentum spectrum of direct photons as measured by the PHENIX Collaboration in Refs. [28, 29]. Furthermore, the PHSD also describes the data on the elliptic flow of inclusive photons [11]. In order to extract the flow of 'direct photons' from the inclusive one, the hadron decay background has to be subtracted. This can be done by two procedures which we describe below.

Procedure 1. We calculate the direct photon $v_{2}$ (in PHSD) by summing up the elliptic flow of the individual channels contributing to the direct photons, using their contributions to the spectrum as the relative $p_{T}$-dependent weights, $w_{i}\left(p_{T}\right)$, i.e.

$$
\begin{gathered}
v_{2}\left(\gamma^{d i r}\right)=\sum_{i} v_{2}\left(\gamma^{i}\right) w_{i}\left(p_{T}\right)=\sum_{i} v_{2}\left(\gamma^{i}\right) N_{i}\left(p_{T}\right) / \sum_{i} N_{i}\left(p_{T}\right), \\
\text { where } i=(q \bar{q} \rightarrow g \gamma, q g \rightarrow q \gamma, \pi \pi / \rho \rightarrow \rho / \pi \gamma, m m \rightarrow m m \gamma, \mathrm{pQCD}) .
\end{gathered}
$$

The index $i$ denotes the binary production channels, both the partonic quark-gluon interaction channels and the meson reactions which cannot be separated presently experimentally by model-independent methods. The direct photon elliptic flow calculated in this way is presented in Fig. 1 (right hand side) by the dashed green line and clearly underestimates the PHENIX data from Ref. $[1,27]$ in line with Refs [2-6].

Procedure 2. The experimental collaboration has extracted the elliptic flow of direct photons $v_{2}\left(\gamma^{d i r}\right)$ from the measured inclusive photon $v_{2}\left(\gamma^{i n c l}\right)$ by subtracting the hadron decay sources $\left(\pi_{0}, \eta\right.$, $\left.\omega, \eta^{\prime}, \phi, a_{1}\right)$ as follows [1]:

$$
v_{2}\left(\gamma^{d i r}\right)=\left(R_{\gamma} v_{2}\left(\gamma^{i n c l}\right)-v_{2}\left(\gamma^{B G}\right)\right) /\left(R_{\gamma}-1\right)=v_{2}\left(\gamma^{B G}\right)+\frac{R_{\gamma}}{R_{\gamma}-1}\left(v_{2}\left(\gamma^{i n c l}\right)-v_{2}\left(\gamma^{B G}\right)\right)
$$



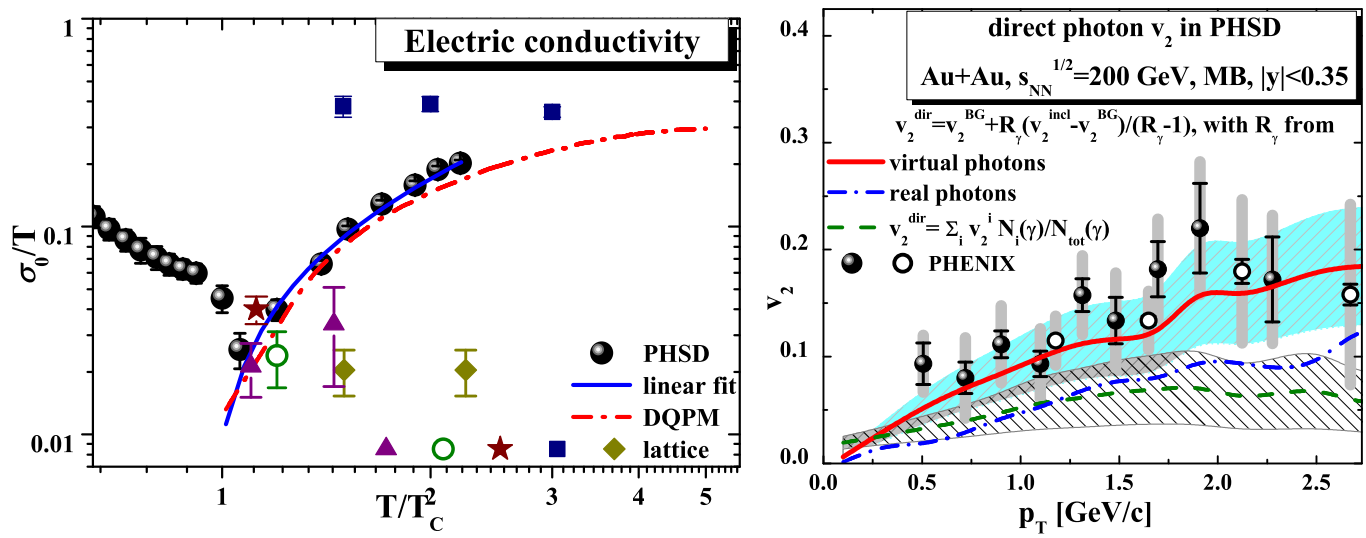

Figure 1. Left hand side: The ratio of electric conductivity to temperature $\sigma_{0} / T$ as a function of the scaled temperature $T / T_{c}\left(T_{c}=158 \mathrm{MeV}\right)$ for hot QCD matter. The full round symbols show the PHSD results, the solid blue line is the linear fit to the PHSD results (above $T_{c}$ ), while the dash-dotted red line gives the corresponding ratio in the relaxation-time approach (employing the DQPM parameters). The various symbols with error bars represent the results from lattice calculations: triangles - quenched QCD results in the continuum limit with Wilson-Clover fermions and renormalized vector currents from Refs. [20], diamonds - quenched QCD with staggered fermions from Ref. [21], squares - earlier work in the quenched QCD with staggered fermions on smaller lattices from Ref. [22], star - quenched SU(2) lattice gauge theory from Ref. [23], open circle - QCD with two dynamical flavors of Wilson-Clover fermions from Ref. [24]. Right hand side: Elliptic flow of direct photons (hadron decays excluded) in the PHSD approach for minimal bias Au+Au collisions at $\sqrt{s_{N N}}=200 \mathrm{GeV}$ in comparison to the data from Refs. [1, 27]. The results from the PHSD are displayed by the solid red line, equation (3), and by the dashed green line, by applying Eq. (2).

where

$$
R_{\gamma}=N^{i n c l} / N^{B G}
$$

denotes the ratio of the inclusive photon yield to that of the "background" (i.e. the photons stemming from the decays of $\pi_{0}, \eta, \omega, \eta^{\prime}, \phi$ and $a_{1}$ mesons), $v_{2}\left(\gamma^{B G}\right)$ is the elliptic flow of the background photons; one can assume $v_{2}\left(\gamma^{B G}\right) \approx v_{2}\left(\pi_{0}\right)$ [11]. The ratio $R_{\gamma}$ was obtained experimentally in Ref. [29] by analyzing the yield of dileptons with high transverse momentum $p_{T}>1 \mathrm{GeV}$ and low invariant mass $M$, the $R_{\gamma}$ values obtained in Ref. [29] for the photon transverse momenta $1<p_{T}<3 \mathrm{GeV}$ are on average $1.2 \pm 0.3$. We recall here that we have studied the dilepton production at the top RHIC energy within the PHSD approach in Ref. [9]. The PHSD results reproduce well the PHENIX and STAR dilepton data differentially in the invariant mass $M$ and transverse momentum $p_{T}$, only underestimating the excess observed by PHENIX at low $M$ and low $p_{T}$. Note, however, that for the relatively high transverse momenta of dileptons $\left(p_{T}>1 \mathrm{GeV}\right)$ the agreement of the PHSD calculations with the PHENIX data is quite good. We obtained $R_{\gamma} \approx 1.2$ by analyzing the yield of dileptons in the invariant mass window $M=0.15-0.3 \mathrm{GeV}$ [11]. Alternatively, we can use the calculated inclusive real photon spectrum to find the ratio $R_{\gamma}=N(\gamma)^{i n c l} / N(\gamma)^{B G}$. In this case we obtained $R_{\gamma} \approx 1.08$ from the real photons in PHSD [11]. The difference between the values of $R_{\gamma}$ - extracted from the dilepton spectra and the real photon spectra - is caused by the fact that in the dilepton mass window $M=0.15-0.3 \mathrm{GeV}$ the background from the pion decays effectively "dies out", while the pion decay contribution is prominent for $M \rightarrow 0$.

Following the procedure of equation (3) in the PHSD, we obtain the red solid line in Fig. 1 (right hand side), if we use the ratio $R_{\gamma}$ from the virtual photons in the invariant mass window $M=0.15-$ 
$0.3 \mathrm{GeV}$, and the blue dash-dotted line, if we use the ratio $R_{\gamma}$ from the calculated real photon spectrum. The two lines differ by about a factor of two. The difference between the two extraction procedures for the direct photon flow $v_{2}\left(p_{T}\right)$ can be attributed to different definitions for the ratio of the inclusive and background photons $\left(R_{\gamma}\right)$.

In conclusion, our findings imply that there is presently no clear signal for 'unconventional physics' (beyond the strong interaction on the partonic and hadronic level) in the photon data from the PHENIX Collaboration within error bars. The strong $v_{2}$ of direct photons - which is comparable to the hadronic $v_{2}$ - in PHSD is attributed to hadronic channels, i.e. to meson binary reactions which are not subtracted in the data. On the other hand, the strong $v_{2}$ of the 'parent' hadrons, in turn, stems from the interactions in the QGP. Accordingly, the presence of the QGP shows up 'indirectly' in the direct photon elliptic flow.

\section{References}

[1] A. Adare et al. (PHENIX Collaboration), Phys. Rev. Lett. 109, 122302 (2012)

[2] R. Chatterjee, E.S. Frodermann, U.W. Heinz, D.K. Srivastava, Phys. Rev. Lett. 96, 202302 (2006)

[3] F.M. Liu, T. Hirano, K. Werner, Y. Zhu, Nucl. Phys. A830, 587C (2009)

[4] M. Dion, C. Gale, S. Jeon, J.F. Paquet, B. Schenke et al., J. Phys. G38, 124138 (2011)

[5] M. Dion, J.F. Paquet, B. Schenke, C. Young, S. Jeon et al., Phys. Rev. C84, 064901 (2011)

[6] R. Chatterjee, H. Holopainen, I. Helenius, T. Renk, K.J. Eskola (2013), arXiv:1305.6443

[7] O. Linnyk, J. Phys. G38, 025105 (2011)

[8] O. Linnyk, E.L. Bratkovskaya, V. Ozvenchuk, W. Cassing, C.M. Ko, Phys. Rev. C84, 054917 (2011)

[9] O. Linnyk, W. Cassing, J. Manninen, E. Bratkovskaya, C. Ko, Phys. Rev. C85, 024910 (2012)

[10] O. Linnyk, W. Cassing, J. Manninen, E. Bratkovskaya, et al., Phys. Rev. C87, 014905 (2013)

[11] O. Linnyk, V. Konchakovski, W. Cassing, E. Bratkovskaya (2013), Phys. Rev. C 88, 034904 (2013)

[12] W. Cassing, E.L. Bratkovskaya, Nucl. Phys. A 831, 215 (2009)

[13] E.L. Bratkovskaya, W. Cassing, V.P. Konchakovski, O. Linnyk, Nucl. Phys. A856, 162 (2011)

[14] W. Cassing, S. Juchem, Nucl. Phys. A 665, 377 (2000), ibid. A 672, 417 (2000)

[15] W. Cassing, Eur. Phys. J. ST 168, 3 (2009)

[16] W. Cassing, E.L. Bratkovskaya, Phys. Rept. 308, 65 (1999)

[17] E.L. Bratkovskaya, W. Cassing, Nucl. Phys. A 619, 413 (1997)

[18] E.L. Bratkovskaya, S.M. Kiselev, G.B. Sharkov, Phys. Rev. C78, 034905 (2008)

[19] W. Cassing, O. Linnyk, T. Steinert, V. Ozvenchuk, Phys.Rev.Lett. 110, 182301 (2013)

[20] H.T. Ding et al., Phys. Rev. D 83, 034504 (2011), H. -T. Ding et al. arXiv:1301.7436 [hep-lat]

[21] G. Aarts et al., Phys. Rev. Lett. 99, 022002 (2007)

[22] S. Gupta, Phys. Lett. B 597, 57 (2004)

[23] P.V. Buividovich et al., Phys. Rev. Lett. 105, 132001 (2010)

[24] B.B. Brandt, A. Francis, H.B. Meyer, H. Wittig (2013), arXiv:1302.0675 [hep-lat]

[25] A. Amato, G. Aarts, C. Allton, P. Giudice, S. Hands et al. (2013), arXiv:1307.6763[hep-lat]

[26] P.B. Arnold, G.D. Moore, L.G. Yaffe, JHEP 0305, 051 (2003)

[27] I. Tserruya (PHENIX Collaboration), Nucl.Phys. A904-905, 225c (2013)

[28] A. Adare et al. (PHENIX), Phys. Rev. C 81, 034911 (2010)

[29] A. Adare et al. (PHENIX Collaboration), Phys. Rev. Lett. 104, 132301 (2010) 